\title{
Tidal effects in quantum field theory
}

\author{
Kays Haddad and Andreas Helset \\ Niels Bohr International Academy and Discovery Center, Niels Bohr Institute, \\ University of Copenhagen, Blegdamsvej 17, Copenhagen DK-2100, Denmark \\ E-mail: kays.haddad@nbi.ku.dk, ahelset@nbi.ku.dk
}

ABSTRACT: We apply the Hilbert series to extend the gravitational action for a scalar field to a complete, non-redundant basis of higher-dimensional operators that is quadratic in the scalars and the Weyl tensor. Such an extension of the action fully describes tidal effects arising from operators involving two powers of the curvature. As an application of this new action, we compute all spinless tidal effects at the leading post-Minkowskian order. This computation is greatly simplified by appealing to the heavy limit, where only a severely constrained set of operators can contribute classically at the one-loop level. Finally, we use this amplitude to derive the $\mathcal{O}\left(G^{2}\right)$ tidal corrections to the Hamiltonian and the scattering angle.

KEYwords: Effective Field Theories, Scattering Amplitudes, Black Holes

ArXiv EPrint: 2008.04920 


\section{Contents}

1 Introduction 1

2 Tidal actions $\quad 3$

2.1 Hilbert series for tidal effects 3

2.2 QED 4

2.3 Gravity 5

3 Tidal effects at the leading-PM order $\quad 6$

$\begin{array}{lll}3.1 & \text { QED } & 7\end{array}$

$\begin{array}{llr}3.2 & \text { Gravity } & 9\end{array}$

$\begin{array}{lll}3.3 & \text { Gravitational Hamiltonian and scattering angle } & 11\end{array}$

$\begin{array}{llr}4 & \text { Summary and outlook } & 13\end{array}$

$\begin{array}{ll}\text { A Hilbert series } & 14\end{array}$

$\begin{array}{ll}\text { B Redundant operators } & 15\end{array}$

$\begin{array}{ll}\text { C Operator basis from an on-shell perspective } & 18\end{array}$

\section{Introduction}

There is a long history of relating scattering amplitudes to conservative two-body classical observables. Traditionally, such approaches have made extensive use of the quantum action of gravity [1], and have been used most commonly to compute non-relativistic classical and quantum corrections to the interaction Hamiltonian [2-6]. Other approaches still have utilized on-shell methods to compute the amplitudes, before producing the interaction Hamiltonian [7-9]. Other than the interaction Hamiltonian, refs. [7, 9, 10] also extracted information about the metric from the two-to-two scattering amplitude.

Even compared to this illustrious record, tremendous progress on this topic in a relatively short time has been inspired by the detection of gravitational waves (GWs) by the LIGO and Virgo collaborations [11]. Developments in this time have by and large focused on the post-Minkowskian (PM) expansion of amplitudes and observables [12, 13]. This has required new tools for the conversion of PM amplitudes to classical quantities such as the interaction Hamiltonian [14-16], the linear and angular impulse and radiated momentum [17, 18], the scattering angle [19-21], and the metric [22]. On the front of the amplitudes themselves, the current state-of-the-art is the third post-Minkowskian (3PM) amplitude for scalar-scalar scattering [23-25] (extended to include tidal effects in ref. [26]). The 3PM amplitude for massless scattering was also computed in ref. [27]. Moreover, 
amplitudes techniques have been used to compute observables in modified theories of gravity $[28,29]$.

There has also been significant progress made on the inclusion of spin effects. The spin- $1 / 2 \times$ spin- $1 / 2$ amplitude was computed up to the second post-Minkowskian order using heavy particle effective theory (HPET) techniques in ref. [30], and was converted to a spinning Hamiltonian as part of the spin-inclusive formalism of ref. [16]. An alternative approach to this amplitude involving the leading singularity was presented in ref. [31]. Making use of the massive on-shell variables of ref. [32], several results including all orders in spin were achieved in refs. [33-39]. Some of the notable results from these works include the interpretation of a Kerr black hole as a minimally-coupled infinite spin particle, the scattering angle at the second post-Minkowskian (2PM) order up to fourth order in spin, an amplitudes interpretation of the Newman-Janis complex deformation of Schwarzchild spacetime, and the full 1PM spinning Hamiltonian. Finally, ref. [40] argued that the scattering of minimally coupled spinning particles minimizes the generated entanglement entropy.

Though a plethora of novel results have been achieved using amplitudes-based approaches, the vast majority of results directly applicable to GW templates have been derived using general relativistic methods. Of particular relevance to this paper are the computations of tidal effects on the binary inspiral problem. In this context, several tools have been applied to the computations of these effects. Two such tools are the post-Newtonian $(\mathrm{PN})$ and PM approximations. In the PN context, tidal moments were first introduced in ref. [41]. Ref. [42] incorporated tidal effects into the effective one-body (EOB) formalism [43], and ref. [44] presented tidal contributions to the binding energy within the EOB. Most recently, tidal effects on the PM scattering angle have been computed in refs. [45, 46]

Up to this point, almost all amplitudes approaches to the binary inspiral problem have ignored finite size and tidal effects. In fact, the recent work of ref. [26] is the first application of amplitudes methods to the calculation of these effects. By focusing on operators quadratic in the Weyl tensor, they computed tidal contributions to spinless amplitudes arising from the electric and magnetic quadrupoles, up to the next-to-leadingPM order $\left(\mathcal{O}\left(G^{3}\right)\right) .{ }^{1}$ Converting their amplitudes to classical observables, they found agreement with results derived from conventional general relativistic methods [42, 44-47].

In this paper, we expand on the work of ref. [26]. Through application of the Hilbert series (see e.g. [48-56]), we obtain a gravitational action comprising all operators quadratic in the Weyl tensor and quadratic in a real scalar field. This action is sufficient to fully describe all spinless tidal contributions to the amplitude at the leading-PM order $\left(\mathcal{O}\left(G^{2}\right)\right)$. Since we are only interested in the classical portion of the amplitude, we exploit the manifest $\hbar$ scaling of the heavy limit of the action to isolate only classically contributing operators [30]. This simplifies the computation, and we are able to straightforwardly produce the full classical tidal integrand at the leading-PM order. Integrating the integrand in principle requires knowledge of the general even-rank triangle integral. However, we are able circumvent this issue since we are simply interested in the leading-in- $\hbar$ portion of the

\footnotetext{
${ }^{1}$ Note that one-loop is the leading order where tidal effects can contribute to conservative dynamics.
} 
integral that is proportional to $S \equiv \pi^{2} / \sqrt{-q^{2}}$. This allows us to find a form of the general even-rank triangle integral that we have explicitly checked up to rank 10, and that was proven in ref. [57] while this paper was in review. Applying this results in the complete leading-PM tidal amplitude. We indeed find the leading-PM contribution of ref. [26] as the leading contribution to our amplitude. We then use our amplitude to derive all leading-PM tidal corrections to the Hamiltonian and the scattering angle, comparing to existing results along the way.

The layout of this paper is as follows: we begin in section 2 by presenting the full tidal actions for electromagnetism and gravity coupled to real scalars at quadratic order in the field strength or the Weyl tensor respectively. We include a brief primer on the Hilbert series in this section, as it is the main tool in our construction. With the tidal actions in hand, section 3 focuses on the computation of tidal contributions to the oneloop amplitudes. The heavy limits of the tidal actions are also presented here. We conclude in section 4.

\section{Tidal actions}

This section is dedicated to the presentation of the tidal actions up to quadratic order in the field strengths or Weyl tensors respectively for QED or gravity coupled to a real scalar. We achieve the complete forms of these actions through application of the Hilbert series. As such, we begin with a brief introduction to the Hilbert series before presenting the results of the series and corresponding tidal actions for QED and then gravity. Technical details about the Hilbert series are postponed to appendix A.

\subsection{Hilbert series for tidal effects}

The Hilbert series uses character orthonormality to count group invariants. It is an important tool for constructing a basis of higher-dimensional operators, and has been applied to the effective-field-theory extension of the Standard Model in refs. [51-54], while ref. [56] also included gravity.

The input for the Hilbert series is the field content and the fields' representations under compact symmetries. The output is the number of invariant operators with a given field content and covariant derivatives. Redundancies coming from integration-by-parts relations and field redefinitions are taken into account.

We first want to construct operators with real scalar fields $\phi$ coupled to photons. The Lorentz group $\mathrm{SO}(1,3)$ is not a compact group, but we can use the Euclidean group $\mathrm{SO}(4) \simeq$ $\mathrm{SU}(2)_{L} \times \mathrm{SU}(2)_{R}$ to find the group invariants. We then work with fields transforming in irreducible representations of $\mathrm{SU}(2)_{L}$ and $\mathrm{SU}(2)_{R}$ built from linear combinations of the field strength $F_{\mu \nu}$ and the dual field strength $\tilde{F}_{\mu \nu}=\frac{1}{2} \epsilon_{\mu \nu \rho \sigma} F^{\rho \sigma}$ :

$$
F_{L / R}^{\mu \nu} \equiv \frac{1}{2}\left(F^{\mu \nu} \pm i \tilde{F}^{\mu \nu}\right)
$$

The characters for $F_{L / R}^{\mu \nu}$ and $\phi$ are the input to the Hilbert series. 
We restrict our attention to the operators with two real scalar fields, two field strengths, and an arbitrary number of covariant derivatives. The output of the Hilbert series $\mathcal{H}_{d}^{F^{2}}$ for mass dimension $d=6+2 n$ is

$$
\mathcal{H}_{6+2 n}^{F^{2}}=\left\lfloor\frac{n+2}{2}\right\rfloor\left(F_{L}^{2} \phi^{2} D^{2 n}+F_{R}^{2} \phi^{2} D^{2 n}\right)+\left\lfloor\frac{n+1}{2}\right\rfloor F_{L} F_{R} \phi^{2} D^{2 n},
$$

where $n \geq 0$ is an integer and $\lfloor x\rfloor$ is the floor function.

Now consider the Hilbert series for two real scalars coupled to gravity. As explained in appendix B, non-redundant operators quadratic in the curvature can be written in terms of the Weyl tensor $C^{\mu \nu \rho \sigma}$. Thus we need only the group characters of

$$
C_{L / R}^{\mu \nu \rho \sigma}=\frac{1}{2}\left(C^{\mu \nu \rho \sigma} \pm i \tilde{C}^{\mu \nu \rho \sigma}\right)
$$

where $\tilde{C}^{\mu \nu \rho \sigma}=\frac{1}{2} \epsilon^{\mu \nu \alpha \beta} C_{\alpha \beta}{ }^{\rho \sigma}$ is the dual to the Weyl tensor. The Hilbert series $\mathcal{H}_{d}^{C^{2}}$ for two real scalar fields, two Weyl tensors, and an arbitrary number of covariant derivatives is

$$
\mathcal{H}_{6+2 n}^{C^{2}}=\left\lfloor\frac{n+2}{2}\right\rfloor\left(C_{L}^{2} \phi^{2} \nabla^{2 n}+C_{R}^{2} \phi^{2} \nabla^{2 n}\right)+\left\lfloor\frac{n}{2}\right\rfloor C_{L} C_{R} \phi^{2} \nabla^{2 n},
$$

for integer $n \geq 0$.

We use the output of the Hilbert series as a guide for constructing a basis of higherdimensional operators which capture all leading-PM tidal effects in electromagnetism and gravity.

\subsection{QED}

The Lagrangian we are after couples a real scalar to photons through operators quadratic in the field strength:

$$
\mathcal{L}_{\mathrm{QED}}=\frac{1}{2}\left(\partial_{\mu} \phi\right)\left(\partial^{\mu} \phi\right)-\frac{m^{2}}{2} \phi^{2}+\Delta \mathcal{L}_{\mathrm{QED}}^{\mathrm{tidal}}
$$

Here $\Delta \mathcal{L}_{\mathrm{QED}}^{\text {tidal }}$ describes the tidal interactions between two real scalars and two field strength tensors. We are interested in using the Hilbert series in eq. (2.2) to construct this contribution at general mass dimension.

Ultimately, there is a freedom in the operator basis we use (see appendix B). We choose a basis that is optimized for the computation of classical amplitudes. Such a basis does not include any structures of the form $D^{\mu} \phi D_{\mu} \phi \mathcal{O}_{F^{2}}$. These can be seen to mix with $\phi^{2} \mathcal{O}_{F^{2}}$ in the heavy limit, hence one could receive contributions to classically-contributing heavy operators from an infinite number of operators in the full action. Furthermore, we will avoid derivative placements that produce any structure that can be removed by a field redefinition; see appendix B for a list of such structures. Accounting for these criteria, we will build our basis out of operators of the following form:

$$
\begin{aligned}
\mathcal{O}_{L L, k}^{(n)} & =\left[D^{\mu_{1} \ldots \mu_{k}} \phi\right]\left[D_{\nu_{1} \ldots \nu_{k}} \phi\right]\left[D_{\mu_{1} \ldots \mu_{k} \alpha_{1} \ldots \alpha_{n-2 k}} F_{L, \rho \sigma}\right]\left[D^{\nu_{1} \ldots \nu_{k} \alpha_{1} \ldots \alpha_{n-2 k}} F_{L}^{\rho \sigma}\right], \\
\mathcal{O}_{R R, k}^{(n)} & =\left[D^{\mu_{1} \ldots \mu_{k}} \phi\right]\left[D_{\nu_{1} \ldots \nu_{k}} \phi\right]\left[D_{\mu_{1} \ldots \mu_{k} \alpha_{1} \ldots \alpha_{n-2 k}} F_{R, \rho \sigma}\right]\left[D^{\nu_{1} \ldots \nu_{k} \alpha_{1} \ldots \alpha_{n-2 k}} F_{R}^{\rho \sigma}\right], \\
\mathcal{O}_{L R, k}^{(n+1)} & =\left[D^{\rho \mu_{1} \ldots \mu_{k}} \phi\right]\left[D_{\sigma \nu_{1} \ldots \nu_{k}} \phi\right]\left[D_{\mu_{1} \ldots \mu_{k} \alpha_{1} \ldots \alpha_{n-2 k}} F_{L, \rho \tau}\right]\left[D^{\nu_{1} \ldots \nu_{k} \alpha_{1} \ldots \alpha_{n-2 k}} F_{R}^{\sigma \tau}\right],
\end{aligned}
$$


where $0 \leq k \leq\lfloor n / 2\rfloor$. This range of $k$ produces the number of operators dictated by the Hilbert series. We have defined $D_{\mu_{1} \ldots \mu_{n}} \equiv D_{\mu_{1}} \ldots D_{\mu_{n}}$.

We would like to construct our action out of the fields $F^{\mu \nu}$ and $\tilde{F}^{\mu \nu}$. To do so we simply replace $F_{L, R}^{\mu \nu}$ in terms of the field strength and its dual. After this replacement the operators above become

$$
\begin{aligned}
\mathcal{O}_{L L, k}^{(n)}= & 2\left[D^{\mu_{1} \ldots \mu_{k}} \phi\right]\left[D_{\nu_{1} \ldots \nu_{k}} \phi\right]\left[D_{\mu_{1} \ldots \mu_{k} \alpha_{1} \ldots \alpha_{n-2 k}} F_{\rho \sigma}\right]\left[D^{\nu_{1} \ldots \nu_{k} \alpha_{1} \ldots \alpha_{n-2 k}} F^{\rho \sigma}\right] \\
& +2 i\left[D^{\mu_{1} \ldots \mu_{k}} \phi\right]\left[D_{\nu_{1} \ldots \nu_{k}} \phi\right]\left[D_{\mu_{1} \ldots \mu_{k} \alpha_{1} \ldots \alpha_{n-2 k}} F_{\rho \sigma}\right]\left[D^{\nu_{1} \ldots \nu_{k} \alpha_{1} \ldots \alpha_{n-2 k}} \tilde{F}^{\rho \sigma}\right], \quad \\
\mathcal{O}_{R R, k}^{(n)}= & 2\left[D^{\mu_{1} \ldots \mu_{k}} \phi\right]\left[D_{\nu_{1} \ldots \nu_{k}} \phi\right]\left[D_{\mu_{1} \ldots \mu_{k} \alpha_{1} \ldots \alpha_{n-2 k}} F_{\rho \sigma}\right]\left[D^{\nu_{1} \ldots \nu_{k} \alpha_{1} \ldots \alpha_{n-2 k}} F^{\rho \sigma}\right] \\
& -2 i\left[D^{\mu_{1} \ldots \mu_{k}} \phi\right]\left[D_{\nu_{1} \ldots \nu_{k}} \phi\right]\left[D_{\mu_{1} \ldots \mu_{k} \alpha_{1} \ldots \alpha_{n-2 k}} F_{\rho \sigma}\right]\left[D^{\nu_{1} \ldots \nu_{k} \alpha_{1} \ldots \alpha_{n-2 k}} \tilde{F}^{\rho \sigma}\right], \quad \\
\mathcal{O}_{L R, k}^{(n+1)}= & 2\left[D^{\rho \mu_{1} \ldots \mu_{k}} \phi\right]\left[D_{\sigma \nu_{1} \ldots \nu_{k}} \phi\right]\left[D_{\mu_{1} \ldots \mu_{k} \alpha_{1} \ldots \alpha_{n-2 k}} F_{\rho \tau}\right]\left[D^{\nu_{1} \ldots \nu_{k} \alpha_{1} \ldots \alpha_{n-2 k}} F^{\sigma \tau}\right] \\
& -\frac{1}{2} \eta^{\rho \sigma}\left[D^{\rho \mu_{1} \ldots \mu_{k}} \phi\right]\left[D_{\sigma \nu_{1} \ldots \nu_{k}} \phi\right]\left[D_{\mu_{1} \ldots \mu_{k} \alpha_{1} \ldots \alpha_{n-2 k}} F_{\rho \tau}\right]\left[D^{\nu_{1} \ldots \nu_{k} \alpha_{1} \ldots \alpha_{n-2 k}} F^{\sigma \tau}\right] .
\end{aligned}
$$

Both operators in eqs. (2.7a) and (2.7b) contain P-odd terms. We are not interested in such effects, so we ignore these operators. Also note that, by integrating by parts twice, the second term in eq. (2.7c) can be reexpressed in terms of other operators already present and terms that can be removed by field redefinitions, up to contributions cubic in the field strength.

All-in-all, there are two generic structures out of which we build the tidal action. The tidal contribution to the action to all mass dimensions is thus

$$
\begin{aligned}
\Delta \mathcal{L}_{\mathrm{QED}}^{\mathrm{tidal}}= & \sum_{n=0}^{\infty} \sum_{k=0}^{N}\left\{a_{k}^{(n)}\left[D^{\mu_{1} \ldots \mu_{k}} \phi\right]\left[D_{\nu_{1} \ldots \nu_{k}} \phi\right]\left[D_{\mu_{1} \ldots \mu_{k} \alpha_{1} \ldots \alpha_{n-2 k}} F_{\rho \sigma}\right]\left[D^{\nu_{1} \ldots \nu_{k} \alpha_{1} \ldots \alpha_{n-2 k}} F^{\rho \sigma}\right]\right. \\
& \left.+b_{k}^{(n+1)}\left[D^{\rho \mu_{1} \ldots \mu_{k}} \phi\right]\left[D_{\sigma \nu_{1} \ldots \nu_{k}} \phi\right]\left[D_{\mu_{1} \ldots \mu_{k} \alpha_{1} \ldots \alpha_{n-2 k}} F_{\rho \tau}\right]\left[D^{\nu_{1} \ldots \nu_{k} \alpha_{1} \ldots \alpha_{n-2 k}} F^{\sigma \tau}\right]\right\}
\end{aligned}
$$

where $N \equiv\lfloor n / 2\rfloor$ and we have introduced the Wilson coefficients $a_{k}^{(n)}$ and $b_{k}^{(n+1)}$. Note that the covariant derivatives acting on the real scalars or field strenghts reduce to partial derivatives. One can easily incorporate P-odd operators into this tidal action by including the same operators as in the first line in eq. (2.8) where one of the field strenghts is replaced by a dual field strength.

\subsection{Gravity}

We repeat the procedure from the previous section, only this time for a real scalar coupled to gravity. The relevant action is

$$
\sqrt{-g} \mathcal{L}_{\mathrm{GR}}=\sqrt{-g}\left[\frac{g^{\mu \nu}}{2}\left(\partial_{\mu} \phi\right)\left(\partial_{\nu} \phi\right)-\frac{m^{2}}{2} \phi^{2}+\Delta \mathcal{L}_{\mathrm{GR}}^{\mathrm{tidal}}\right] .
$$

We will find the form of the tidal contribution at general mass dimension using the Hilbert series in eq. (2.4). 
The optimal basis for our purposes satisfies the same criteria as in the previous section. As such, our basis comprises operators of the form

$$
\begin{aligned}
\mathcal{O}_{L L, k}^{(n)} & =\left[\nabla^{\mu_{1} \ldots \mu_{k}} \phi\right]\left[\nabla_{\nu_{1} \ldots \nu_{k}} \phi\right]\left[\nabla_{\mu_{1} \ldots \mu_{k} \alpha_{1} \ldots \alpha_{n-2 k}} C_{L, \rho \sigma \alpha \beta}\right]\left[\nabla^{\nu_{1} \ldots \nu_{k} \alpha_{1} \ldots \alpha_{n-2 k}} C_{L}^{\rho \sigma \alpha \beta}\right], \\
\mathcal{O}_{R R, k}^{(n)} & =\left[\nabla^{\mu_{1} \ldots \mu_{k}} \phi\right]\left[\nabla_{\nu_{1} \ldots \nu_{k}} \phi\right]\left[\nabla_{\mu_{1} \ldots \mu_{k} \alpha_{1} \ldots \alpha_{n-2 k}} C_{R, \rho \sigma \alpha \beta}\right]\left[\nabla^{\nu_{1} \ldots \nu_{k} \alpha_{1} \ldots \alpha_{n-2 k}} C_{R}^{\rho \sigma \alpha \beta}\right], \\
\mathcal{O}_{L R, k}^{(n+2)} & =\left[\nabla^{\rho \sigma \mu_{1} \ldots \mu_{k}} \phi\right]\left[\nabla_{\alpha \beta \nu_{1} \ldots \nu_{k}} \phi\right]\left[\nabla_{\mu_{1} \ldots \mu_{k} \alpha_{1} \ldots \alpha_{n-2 k}} C_{L, \lambda \rho \tau \sigma}\right]\left[\nabla^{\nu_{1} \ldots \nu_{k} \alpha_{1} \ldots \alpha_{n-2 k}} C_{R}^{\lambda \alpha \tau \beta}\right] .
\end{aligned}
$$

We introduced the shorthand notation $\nabla_{\mu_{1} \ldots \mu_{n}}=\nabla_{\mu_{1}} \ldots \nabla_{\mu_{n}}$. In this case as well $k$ is in the range $0 \leq k \leq N$.

These operators can be expressed in terms of the Weyl tensor and its dual. The exact same procedure as in the QED case, along with covariant conservation of the Levi-Civita tensor [58], results in only two forms of operators comprising the basis, modulo P-odd operators. The tidal contribution to the action is thus

$$
\begin{aligned}
& \Delta \mathcal{L}_{\mathrm{GR}}^{\mathrm{tidal}}= \\
& \quad \sum_{n=0}^{\infty} \sum_{k=0}^{N}\left\{c_{k}^{(n)}\left[\nabla^{\mu_{1} \ldots \mu_{k}} \phi\right]\left[\nabla_{\nu_{1} \ldots \nu_{k}} \phi\right]\left[\nabla_{\mu_{1} \ldots \mu_{k} \alpha_{1} \ldots \alpha_{n-2 k}} C_{\rho \sigma \alpha \beta}\right]\left[\nabla^{\nu_{1} \ldots \nu_{k} \alpha_{1} \ldots \alpha_{n-2 k}} C^{\rho \sigma \alpha \beta}\right]\right. \\
& \left.\quad+d_{k}^{(n+2)}\left[\nabla^{\rho \sigma \mu_{1} \ldots \mu_{k}} \phi\right]\left[\nabla_{\alpha \beta \nu_{1} \ldots \nu_{k}} \phi\right]\left[\nabla_{\mu_{1} \ldots \mu_{k} \alpha_{1} \ldots \alpha_{n-2 k}} C_{\lambda \rho \tau \sigma}\right]\left[\nabla^{\nu_{1} \ldots \nu_{k} \alpha_{1} \ldots \alpha_{n-2 k}} C^{\lambda \alpha \tau \beta}\right]\right\}
\end{aligned}
$$

to all mass dimensions. The coefficients $c_{k}^{(n)}$ and $d_{k}^{(n+2)}$ are the Wilson coefficients for the action. Again, the P-odd operators which could be added to the basis take the same form as the first line in eq. (2.13) with one of the Weyl tensors replaced by a dual Weyl tensor.

\section{Tidal effects at the leading-PM order}

The actions in eqs. (2.8) and (2.13) describe all tidal effects that can arise from terms quadratic in the electromagnetic field strength and the curvature, respectively. In fact, these actions are sufficient for describing all tidal effects at the one-loop order, which corresponds to the leading-PM order in the case of gravity. We present in this section the full classical one-loop tidal contributions to both electromagnetic and gravitational amplitudes. Since we are exclusively interested in classical contributions, we can take advantage of the heavy limits of these actions to identify the only operators which contribute classically at this loop order [30]. This results in significant simplifications to the Feynman rules and the loop integrals involved.

We follow the method in refs. [30, 59-61] to take the heavy limit of real scalars. Namely, we apply the field redefinition

$$
\phi \rightarrow \frac{1}{\sqrt{2 m}}\left(e^{-i m v \cdot x} \chi+e^{i m v \cdot x} \chi^{*}\right)
$$

and drop quickly oscillating terms. Furthermore, by counting the powers of $\hbar$ associated with each operator, and given that the triangle diagram in figure 1 is the only topology 


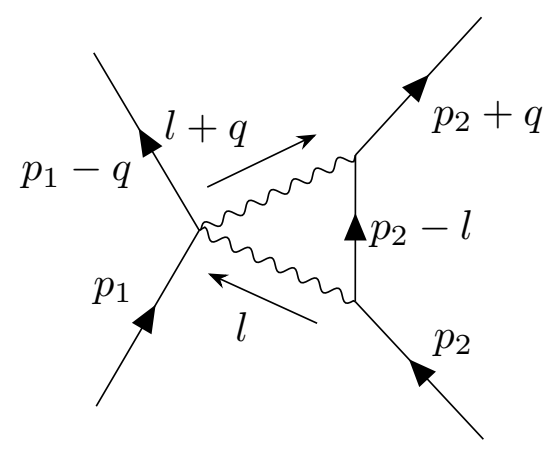

Figure 1. The only topology contributing classical tidal effects at one loop. The tidal effects of particle 1 are probed. The wavy lines represent either photons or gravitons.

of interest at the one-loop level, we only need the operators at leading order in the $1 / \mathrm{m}$ expansion.

We present first the heavy limit of the electromagnetic tidal action, as well as the full classical one-loop tidal contribution to the electromagnetic amplitude, before moving on to the case of gravity. We have normalized all amplitudes by multiplying by $4 m_{1} m_{2}$. This compensates for the normalization in eq. (3.1). ${ }^{2}$

\subsection{QED}

Beginning with the Lagrangian in eq. (2.5), we apply the field redefinition in eq. (3.1) to obtain

$$
\mathcal{L}_{\mathrm{HQET}}=\chi^{*} i v \cdot \partial \chi+\Delta \mathcal{L}_{\mathrm{HQET}}^{\mathrm{tidal}}+\ldots
$$

where

$$
\begin{aligned}
& \Delta \mathcal{L}_{\mathrm{HQET}}^{\mathrm{tidal}}= \\
& \quad \sum_{n=0}^{\infty} \sum_{k=0}^{N}\left\{a_{k}^{(n)} m^{2 k-1}\left[v^{\mu_{1} \ldots \mu_{k}} \chi^{*}\right]\left[v_{\nu_{1} \ldots \nu_{k}} \chi\right]\left[D_{\mu_{1} \ldots \mu_{k} \alpha_{1} \ldots \alpha_{n-2 k}} F_{\rho \sigma}\right]\left[D^{\nu_{1} \ldots \nu_{k} \alpha_{1} \ldots \alpha_{n-2 k}} F^{\rho \sigma}\right]\right. \\
& \left.\quad+b_{k}^{(n+1)} m^{2 k+1}\left[v^{\rho \mu_{1} \ldots \mu_{k}} \chi^{*}\right]\left[v_{\sigma \nu_{1} \ldots \nu_{k}} \chi\right]\left[D_{\mu_{1} \ldots \mu_{k} \alpha_{1} \ldots \alpha_{n-2 k}} F_{\rho \tau}\right]\left[D^{\nu_{1} \ldots \nu_{k} \alpha_{1} \ldots \alpha_{n-2 k}} F^{\sigma \tau}\right]\right\}+\ldots
\end{aligned}
$$

We have defined $v_{\mu_{1} \ldots \mu_{n}}=v_{\mu_{1}} \ldots v_{\mu_{n}}$. In these equations, dots represent operators scaling with higher powers of $\hbar$. We can ignore these operators as the computation of classical effects at the one-loop level only requires contributions from the leading-in- $\hbar$ operators.

At this point there is an apparent contradiction in the tidal operators we have claimed to be leading in $\hbar$. Increasing $n$ or decreasing $k$ at fixed $n$ increases the number of derivatives acting on the photon field, thereby increasing powers of $\hbar$ in the resulting contributions to amplitudes [17]. However, the derivative structure of the subleading tidal terms in the worldline action $[44,45,47]$ suggests that we are right to keep these terms. Therefore, much like in the case of spin effects where the spin vector absorbs a power of $\hbar$ [18], we propose

\footnotetext{
${ }^{2}$ More precisely, this factor is the leading-in- $\hbar$ portion of the heavy scalar external states in momentum space [62].
} 
that the tidal coefficients must scale with $\hbar$ to absorb the factors from the operators. The scalings that cancel those of the operators in eq. (3.2) are

$$
\begin{aligned}
a_{k}^{(n)} & \sim \hbar^{-2 n+2 k-2}, \\
b_{k}^{(n+1)} & \sim \hbar^{-2 n+2 k-2} .
\end{aligned}
$$

As in the case of spin, this scaling is necessary to make contact between portions of the amplitude and classical quantities.

We proceed now to use the heavy action to compute the classical one-loop tidal amplitude. We let particle $i$ have momentum $p_{i}=m_{i} v_{i}+k_{i}$, where we have applied the usual heavy-particle decomposition of the momentum. Note that we cannot generate three-point vertices with a photon and two real scalars, so we take particle 2 to be complex in this context, i.e. particle 2 obeys the action given by eq. (B.3) in ref. [30]. The portion of the leading-PM amplitude involving only the $k=0$ terms in eq. (3.2) is

$$
\Delta \mathcal{A}_{2}^{k=0}=-\frac{e^{2}}{\pi^{2}} S m_{2} \sum_{n=0}^{\infty}(-1)^{n}\left(\frac{q^{2}}{2}\right)^{n+1}\left[a_{0}^{(n)}+\frac{m_{1}^{2}}{8} b_{0}^{(n+1)}\left(3 \omega^{2}+1\right)\right] .
$$

We have defined $S \equiv \pi^{2} / \sqrt{-q^{2}}$ and $\omega \equiv v_{1} \cdot v_{2}$. The notation $\Delta \mathcal{A}$ denotes an electromagnetic amplitude linear in the tidal coefficients in eq. (3.2b).

Let's now extend this result to general $k$. The unintegrated form of the amplitude is

$$
\begin{aligned}
\Delta \mathcal{A}_{2}=-8 i e^{2} m_{2} \sum_{n=0}^{\infty} \sum_{k=0}^{N}( & -1)^{n} m_{1}^{2 k}\left(\frac{q^{2}}{2}\right)^{n-2 k}\left[2\left(\frac{q^{2}}{2}\right) a_{k}^{(n)} v_{1 \mu_{1} \ldots \mu_{2 k}} \mathcal{I}_{\triangleleft}^{\mu_{1} \ldots \mu_{2 k}}\right. \\
& \left.+m_{1}^{2} b_{k}^{(n+1)}\left(\omega^{2} \frac{q^{2}}{2} v_{\mu_{1} \ldots \mu_{2 k}} \mathcal{I}_{\triangleleft}^{\mu_{1} \ldots \mu_{2 k}}+v_{\mu_{1} \ldots \mu_{2 k+2}} \mathcal{I}_{\triangleleft}^{\mu_{1} \ldots \mu_{2 k+2}}\right)\right] .
\end{aligned}
$$

To integrate the general $k$ amplitude, we need knowledge of integrals of the form

$$
v_{1 \mu_{1} \ldots \mu_{2 k}} \mathcal{I}_{\triangleleft}^{\mu_{1} \ldots \mu_{2 k}}=\int \frac{d^{4} l}{(2 \pi)^{4}} \frac{\left(v_{1} \cdot l\right)^{2 k}}{l^{2}(l+q)^{2}\left(-v_{2} \cdot l\right)} .
$$

This task is simplified since we in fact only need the portion of this integral proportional to the non-analytic structure $S$, and even then only the leading-in- $\hbar$ contribution to this portion. We observe the following pattern for the portion of the integral we are interested in:

$$
v_{1 \mu_{1} \ldots \mu_{2 k}} \mathcal{I}_{\triangleleft}^{\mu_{1} \ldots \mu_{2 k}}=\frac{\left(\frac{1}{2}\right)_{k}}{4^{k}(1)_{k}}\left(\omega^{2}-1\right)^{k} q^{2 k} \mathcal{I}_{\triangleleft}+\mathcal{O}\left(\hbar^{2 k}\right),
$$

where $(a)_{b}$ is the Pochhammer symbol and

$$
\mathcal{I}_{\triangleleft}=\int \frac{d^{4} l}{(2 \pi)^{4}} \frac{1}{l^{2}(l+q)^{2}\left(-v_{2} \cdot l\right)} .
$$

Note that, since the scalar triangle integral scales as $\hbar^{-1}$, the leading term in eq. (3.7) scales as $\hbar^{2 k-1}$. We have explicitly checked eq. (3.7) up to $2 k=10$ using the Passarino-Veltman reduction [63]. Equation (3.7) was proven in ref. [57] while this paper was in review. 
Armed with eq. (3.7), we compute a result for general $n, k$;

$$
\begin{aligned}
\Delta \mathcal{A}_{2}=-\frac{e^{2} S m_{2}}{\pi^{2}} \sum_{n=0}^{\infty} & \sum_{k=0}^{N}(-1)^{n}\left(\frac{q^{2}}{2}\right)^{n-k+1} m_{1}^{2 k}\left(\omega^{2}-1\right)^{k} \\
& \times\left\{a_{k}^{(n)} \frac{\left(\frac{1}{2}\right)_{k}}{2^{k}(1)_{k}}+\frac{1}{2} m_{1}^{2} b_{k}^{(n+1)}\left[\frac{\left(\frac{1}{2}\right)_{k}}{(1)_{k}} \omega^{2}-\frac{\left(\frac{1}{2}\right)_{k+1}}{2(1)_{k+1}}\left(\omega^{2}-1\right)\right]\right\} .
\end{aligned}
$$

We can reorganize the sums to make the dependence on $q^{2}$ more transparent:

$$
\Delta \mathcal{A}_{2}=\sum_{i=0}^{\infty} \frac{e^{2}}{\pi^{2}}\left(-\frac{q^{2}}{2}\right)^{i+1} \operatorname{Sm}_{2} f_{i}(\omega)
$$

where

$$
f_{i}(\omega) \equiv \sum_{k=0}^{i} \frac{\left(\frac{1}{2}\right)_{k}}{2^{k}(1)_{k}}\left(1-\omega^{2}\right)^{k}\left[m_{1}^{2 k} a_{k}^{(i+k)}+\frac{2^{k}}{8(k+1)} m_{1}^{2 k+2} b_{k}^{(i+k+1)}\left[(2 k+3) \omega^{2}+(2 k+1)\right]\right],
$$

after some algebraic simplification of the Pochhammer symbols.

\subsection{Gravity}

We turn now to the leading-PM gravitational tidal amplitude. Once again the first step is to find the action describing a heavy scalar. Beginning with the Lagrangian in eq. (2.9), we apply the field redefinition in eq. (3.1) to obtain

$$
\sqrt{-g} \mathcal{L}_{\mathrm{HBET}}=\sqrt{-g}\left[\frac{1}{2} m\left(g^{\mu \nu} v_{\mu} v_{\nu}-1\right) \chi^{*} \chi+\chi^{*} i v \cdot \partial \chi+\Delta \mathcal{L}_{\mathrm{HBET}}^{\mathrm{tidal}}+\ldots\right],
$$

where

$$
\begin{aligned}
& \Delta \mathcal{L}_{\mathrm{HBET}}^{\mathrm{tidal}}= \\
& \quad \sum_{n=0}^{\infty} \sum_{k=0}^{N}\left\{c_{k}^{(n)} m^{2 k-1}\left[v^{\mu_{1} \ldots \mu_{k}} \chi^{*}\right]\left[v_{\nu_{1} \ldots \nu_{k}} \chi\right]\left[\nabla_{\mu_{1} \ldots \mu_{k} \alpha_{1} \ldots \alpha_{n-2 k}} C_{\rho \sigma \alpha \beta}\right]\left[\nabla^{\nu_{1} \ldots \nu_{k} \alpha_{1} \ldots \alpha_{n-2 k}} C^{\rho \sigma \alpha \beta}\right]\right. \\
& \left.\quad+d_{k}^{(n+2)} m^{2 k+3}\left[v^{\rho \sigma \mu_{1} \ldots \mu_{k}} \chi^{*}\right]\left[v_{\alpha \beta \nu_{1} \ldots \nu_{k}} \chi\right]\left[\nabla_{\mu_{1} \ldots \mu_{k} \alpha_{1} \ldots \alpha_{n-2 k}} C_{\lambda \rho \tau \sigma}\right]\left[\nabla^{\nu_{1} \ldots \nu_{2} \alpha_{1} \ldots \alpha_{n-2 k}} C^{\lambda \alpha \tau \beta}\right]\right\} \\
& \quad+\ldots
\end{aligned}
$$

In these equations, dots represent operators scaling with higher powers of $\hbar$. Note that we must keep the term $\chi^{*} i v \cdot \partial \chi$ in the action even though it is subleading in $m$ since it is the kinetic term for the heavy scalar. Following the arguments in section 3.1, we propose the following $\hbar$-scaling of the gravitational tidal coefficients:

$$
\begin{aligned}
c_{k}^{(n)} & \sim \hbar^{-2 n+2 k-4}, \\
d_{k}^{(n+2)} & \sim \hbar^{-2 n+2 k-4} .
\end{aligned}
$$


First we reproduce the leading-PM amplitude from ref. [26]. We need only the operators with $n=k=0$ for this task. Thus the amplitude for the leading tidal effect is

$$
\Delta \mathcal{M}_{2}^{n=k=0}=G^{2} q^{4} S m_{2}^{3}\left[16 c_{0}^{(0)}+\frac{m_{1}^{4}}{8} d_{0}^{(2)}\left(35 \omega^{4}-30 \omega^{2}+11\right)\right] .
$$

This agrees with ref. [26] with the identification $c_{0}^{(0)} \rightarrow \lambda / 4$ and $d_{0}^{(2)} \rightarrow \eta /\left(4 m_{1}^{4}\right)$. Here $\Delta \mathcal{M}$ is a gravitational amplitude linear in the tidal coefficients in eq. (3.12b).

We easily extend this result by including terms at all orders in $n$ and with $k=0$ :

$$
\Delta \mathcal{M}_{2}^{k=0}=4 G^{2} S m_{2}^{3} \sum_{n=0}^{\infty}(-1)^{n}\left(\frac{q^{2}}{2}\right)^{n+2}\left[16 c_{0}^{(n)}+\frac{m_{1}^{4}}{8} d_{0}^{(n+2)}\left(35 \omega^{4}-30 \omega^{2}+11\right)\right] .
$$

The result for general $k$ depends on integrals of the form in eq. (3.6). Specifically, the amplitude in terms of these integrals is

$$
\begin{aligned}
\Delta \mathcal{M}_{2}= & 512 i \pi^{2} G^{2} m_{2}^{3} \sum_{n=0}^{\infty} \sum_{k=0}^{N}(-1)^{n} m_{1}^{2 k}\left(\frac{q^{2}}{2}\right)^{n-2 k+2}\left\{2 c_{k}^{(n)} v_{\mu_{1} \ldots \mu_{2 k}} \mathcal{I}_{\triangleleft}^{\mu_{1} \ldots \mu_{2 k}}\right. \\
& +m_{1}^{4} d_{k}^{(n)}\left[q^{4}\left(1-2 \omega^{2}\right)^{2} v_{\mu_{1} \ldots \mu_{2 k}} \mathcal{I}_{\triangleleft}^{\mu_{1} \ldots \mu_{2 k}}+4 q^{2}\left(1-4 \omega^{2}\right) v_{\mu_{1} \ldots \mu_{2 k+2}} \mathcal{I}_{\triangleleft}^{\mu_{1} \ldots \mu_{2 k+2}}\right. \\
& \left.\left.+8 v_{\mu_{1} \ldots \mu_{2 k+4}} \mathcal{I}_{\triangleleft}^{\mu_{1} \ldots \mu_{2 k+4}}\right]\right\} .
\end{aligned}
$$

We can integrate this using eq. (3.7) to obtain the result for all $n, k$ :

$$
\begin{aligned}
& \Delta \mathcal{M}_{2}=4 G^{2} m_{2}^{3} S \sum_{n=0}^{\infty} \sum_{k=0}^{N}(-1)^{n} m_{1}^{2 k}\left(\omega^{2}-1\right)^{k}\left(\frac{q^{2}}{2}\right)^{n-k+2}\left\{16 c_{k}^{(n)} \frac{\left(\frac{1}{2}\right)_{k}}{2^{k}(1)_{k}}\right. \\
& \left.+m_{1}^{4} d_{k}^{(n+2)}\left[\left(1-2 \omega^{2}\right)^{2} \frac{\left(\frac{1}{2}\right)_{k}}{2^{k-1}(1)_{k}}+\left(1-4 \omega^{2}\right)\left(\omega^{2}-1\right) \frac{\left(\frac{1}{2}\right)_{k+1}}{2^{k-1}(1)_{k+1}}+\left(\omega^{2}-1\right)^{2} \frac{\left(\frac{1}{2}\right)_{k+2}}{2^{k}(1)_{k+2}}\right]\right\} .
\end{aligned}
$$

A suggestive structure arises when $k \neq 0$ : each contribution is proportional to the factor $\left(\omega^{2}-1\right)^{k}$. This factor is small in the PN limit, thus we can see already from the PM amplitude level that the corresponding operators must be subleading in the PN limit, in agreement with the constructions in refs. [44, 45, 47]. In fact, this squares perfectly with principles from classical gravitational effective field theories (EFTs). Terms with $k \neq 0$ involve derivatives of the Weyl tensor of the form $v \cdot \nabla$. These reduce to time derivatives in the PN limit, which are subleading compared to spatial derivatives [64].

Once again, we reorganize the sums in powers of the transfer momentum. The advantage of doing so is that contributions are grouped by their significance to observables. We find

$$
\Delta \mathcal{M}_{2}=4 G^{2} S m_{2}^{3} \sum_{i=0}^{\infty}\left(-\frac{q^{2}}{2}\right)^{i+2} g_{i}(\omega)
$$


where after some simplification

$$
\begin{aligned}
g_{i}(\omega) \equiv \sum_{k=0}^{i} & \frac{(-1)^{k}\left(\frac{1}{2}\right)_{k}}{2^{k}(1)_{k}}\left(\omega^{2}-1\right)^{k}\left[16 m_{1}^{2 k} c_{k}^{(i+k)}\right. \\
& \left.+\frac{m_{1}^{2 k+4} d_{k}^{(i+k+2)}}{4(k+2)(k+1)}\left[(2 k+5)(2 k+7) \omega^{4}-6(2 k+5) \omega^{2}+\left(4 k^{2}+12 k+11\right)\right]\right] .
\end{aligned}
$$

The amplitude is now presented in an optimal form for conversion to the Hamiltonian or scattering angle. We present these quantities in the next section, and defer comparison of this result with the literature until then.

In this section we have only computed the tidal contribution of particle 1 to the amplitude. If one is interested in the tidal effects from both particles at this order, one must simply symmetrize the results here in the particle labels.

\subsection{Gravitational Hamiltonian and scattering angle}

We use now our leading-PM amplitude in eq. (3.18) to compute the full leading-PM tidal corrections to the Hamiltonian and the scattering angle. Beginning with the Hamiltonian, there are two ways we may proceed. The first is to match to the EFT of ref. [14], and the second is through the Lippmann-Schwinger equation [29]. As we are working to linear order in the tidal coefficients, there will be no contributions from the Born iteration, so we work here with the latter formulation. The Hamiltonian as a function of the center-of-mass momentum and the separation between the bodies is given by

$$
H(\mathbf{p}, \mathbf{r})=\sum_{n=1,2} \sqrt{\mathbf{p}^{2}+m_{i}^{2}}+V(\mathbf{p}, \mathbf{r})+\Delta V(\mathbf{p}, \mathbf{r}) .
$$

Here $V(\mathbf{p}, \mathbf{r})$ is the point particle potential and can be found up to $3 \mathrm{PM}$ order in refs. [23, 24]. $\Delta V(\mathbf{p}, \mathbf{r})$ incorporates tidal corrections. At the order to which we have worked, these tidal corrections are simply the Fourier transform of the leading-PM amplitude in the center-of-mass frame:

$$
\Delta V(\mathbf{p}, \mathbf{r})=-\int \frac{d^{3} \mathbf{q}}{(2 \pi)^{3}} e^{-i \mathbf{q} \cdot \mathbf{r}} \Delta \mathcal{M}_{2}(p, q)
$$

In this frame the transfer momentum becomes $q^{\mu}=(0, \mathbf{q})$, so $q^{2}=-\mathbf{q}^{2}$. Substituting now eq. (3.18) into this after incorporating the non-relativistic normalization $1 / 4 E_{1} E_{2}$,

$$
\Delta V(\mathbf{p}, \mathbf{r})=-\frac{G^{2} m_{2}^{3}}{E^{2} \xi} \sum_{i=0}^{\infty} \frac{(-1)^{i}(2 i+4) !}{2^{i+3} r^{2 i+6}} g_{i}(\omega),
$$

where $E \equiv E_{1}+E_{2}$ is the total energy in the center-of-mass frame and $\xi \equiv E_{1} E_{2} / E^{2}$. The $i=0$ term is in exact agreement with eq. (10) of ref. [26].

With this in hand we can compute the scattering angle using the method of ref. [20]. Note that $V_{\text {eff }}$ in ref. [20] is related to the potential in position space by $V_{\text {eff }}=2 E \xi \Delta V .^{3}$

\footnotetext{
${ }^{3}$ We thank Andrea Cristofoli for pointing this out.
} 
Accounting for this, the scattering angle is

$$
\Delta \chi=\frac{G^{2} m_{2}^{3}}{E} \sum_{i=0}^{\infty} \frac{(-1)^{i}(2 i+4) !(i+3)}{2^{i+2} p_{\infty}^{2} b^{2(i+3)}} \frac{\sqrt{\pi} \Gamma\left(i+\frac{7}{2}\right)}{\Gamma(i+4)} g_{i}(\omega),
$$

where $b$ is the impact parameter and $p_{\infty}=|\mathbf{p}|$, the magnitude of the center-of-mass threemomentum. Evaluating this at $i=0$ and noting that $p_{\infty} b=J$, the angular momentum, we find exact agreement with the $\mathcal{O}\left(J^{-6}\right)$ portion of eq. (13) in ref. [26]. This also agrees with ref. [45] upon converting to their notation and matching Wilson coefficients:

$$
\begin{aligned}
p_{\infty} & \rightarrow \frac{m_{1} m_{2}}{E} p_{\infty}, \quad J \rightarrow G m_{1} m_{2} j, \\
c_{0}^{(0)} & \rightarrow-\frac{1}{12} m \sigma^{(2)}, \quad d_{0}^{(2)} \rightarrow \frac{1}{4 m^{3}}\left(\mu^{(2)}+\frac{8}{3} \sigma^{(2)}\right) .
\end{aligned}
$$

A similar notation conversion along with the Wilson coefficient map in eq. (3.24) also produces agreement with the sum of eqs. (5.5) and (5.6) of ref. [46]. We remark that the Wilson coefficient matching in eq. (3.24) is equivalent to the matching of ref. [26]. Moreover, this matching can be seen directly from the level of the heavy action: it is the condition that equates the $n=0, k=0$ portion of the heavy tidal action eq. (3.12b) with the $l=2$ term of the classical worldline action in ref. [47], up to factors of $\chi^{*} \chi$.

To check the $i=1$ term we have repeated the calculation starting from the worldline action of ref. [45], promoting each term to a quantum-field-theory operator, and multiplying by $\chi^{*} \chi$. Doing so we find the following matching conditions on the Wilson coefficients of the two operator bases:

$$
\begin{aligned}
c_{0}^{(1)} & \rightarrow-\frac{1}{32} m_{1} \sigma^{(3)}, \\
c_{1}^{(2)} & \rightarrow \frac{1}{144 m_{1}}\left(-\mu^{(3)}-12 \sigma^{\prime(2)}+\frac{9}{2} \sigma^{(3)}\right), \\
d_{0}^{(3)} & \rightarrow \frac{1}{12 m_{1}^{3}}\left(\mu^{(3)}+3 \sigma^{(3)}\right), \\
d_{1}^{(4)} & \rightarrow \frac{1}{36 m_{1}^{5}}\left(9 \mu^{(2)}-\mu^{(3)}+24 \sigma^{(2)}-3 \sigma^{(3)}\right) .
\end{aligned}
$$

This mapping is also consistent with the form factors in eq. (4.39) of ref. [45], reproducing the same $\omega$ structure in $g_{i}(\omega)$ as in the form factors. ${ }^{4}$ Note, however, that this mapping is only appropriate up to overall constants when comparing to the form factors, as we are comparing different quantities.

As a final check on the Wilson coefficient matching conditions, we computed the factorizable portion of the tree-level $3 \rightarrow 3$ amplitude at linear order in the tidal coefficients. Matching the amplitudes computed from both bases, we indeed find again the matching conditions in eqs. (3.24) and (3.25).

\footnotetext{
${ }^{4}$ Note that $\omega$ in our notation is equivalent to $\gamma$ in the notation of ref. [45].
} 


\section{Summary and outlook}

While the application of scattering amplitudes to the binary point-particle inspiral problem has seen much progress in recent years, the description of finite size and tidal effects is a novel and exciting development. We have demonstrated the applicability of powerful EFT tools to this problem. Namely, through the Hilbert series we have been able to write down an action which includes all possible operators involving two real scalars and two Weyl tensors. These operators represent the leading-PM tidal effects, and the action they compose is sufficient to describe all tidal contributions to the $2 \mathrm{PM}$ amplitude for scalarscalar scattering.

The computation of this amplitude was easily performed by taking the heavy limit of the tidal action and isolating only those operators with the correct $\hbar$ scaling to contribute classically. A subtlety arose in this identification of classically contributing operators: operators with an increasing number of derivatives acting on the Weyl tensors would have to be considered classical. This runs counter to the wisdom that more derivatives produce more powers of $\hbar$. To resolve this tension, we proposed that the Wilson coefficients of the action must themselves scale with compensating powers of $\hbar$, analogously to the absorption of $\hbar$ by the spin vector. We presented the unintegrated form of the leading-PM amplitude, and integrated it using the form of the rank- $2 k$ triangle integral in eq. (3.7). We found agreement where our amplitude has overlap with existing results. The amplitudes were then converted into a Hamiltonian and scattering angle, and once again we found agreement with known results.

There are two obvious extensions to this work. The first is the inclusion of tidal effects from operators with higher powers of the Weyl tensor. An operator involving $n$ powers of the Weyl tensor contributes to vertices with $n$ or more gravitons and two matter lines, and thus contributes to conservative dynamics starting at the $n \mathrm{PM}$ order. Second is the inclusion of spin effects. This point is perhaps the more pressing of the two, since tidal effects for objects of large enough spin may also have implications for the Compton amplitude. The gravitational Compton amplitude acquires a spurious pole for matter with spin $s \geq 2$ [32], an occurrence which is believed to derive from the necessarily composite nature of particles with large spin. If this is true then it is natural to expect that the inclusion of tidal effects may aid in remedying this non-locality. Both of these avenues can be pursued using the same Hilbert series methods we have employed here. We leave these ideas for future research.

\section{Acknowledgments}

We thank Clifford Cheung, Andrea Cristofoli, Poul H. Damgaard, Michèle Levi, Mikhail Solon, and Matt von Hippel for related discussions. We also thank Clifford Cheung and Mikhail Solon for comments on this manuscript. This project has received funding from the European Union's Horizon 2020 research and innovation programme under the Marie Skłodowska-Curie grant agreement No. 764850 "SAGEX". The work of A.H. was supported in part by the Danish Research Foundation (DNRF91) and the Carlsberg Foundation. 


\section{A Hilbert series}

Below we list the mathematical details we used in the construction of the Hilbert series for tidal effects. For a detailed account of the Hilbert series, see e.g. refs. [50-56].

The Hilbert series $\mathcal{H}$ for a given field content $\phi$ is the contour integral of the plethystic exponential: ${ }^{5}$

$$
\mathcal{H}=\int d \mu \frac{1}{P} \mathrm{PE}\left[\chi_{\phi}\right]
$$

where the plethystic exponential (PE) generates all symmetric (antisymmetric) tensor products of the representations of the bosonic (fermionic) field content. The factor $1 / P$ removes a total derivative, where the momentum generating function $P$ is defined below in eq. (A.15). The plethystic exponential takes the form

$$
\mathrm{PE}_{\phi}=\exp \left[\sum_{r=0}^{\infty} z^{r+1} \frac{\phi^{r}}{r \mathcal{D}^{r \Delta_{\phi}}} \chi_{\phi}\left(x_{1}^{r}, \ldots, x_{k}^{r}\right)\right],
$$

where $\Delta_{\phi}$ is the mass dimension of $\phi$ and $z= \pm 1$ when $\phi$ is a boson/fermion, respectively. Here $\chi_{\phi}$ is the character of the representation of $\phi$. When we consider several fields, we simply multiply their plethystic exponentials.

We are using the Hilbert series to generate operators with neutral scalars, photons, and gravitons. Thus we need their respective conformal representations:

$$
\begin{aligned}
\chi_{\phi} & =\chi_{[1,(0,0)]}(\mathcal{D} ; \alpha, \beta), \\
\chi_{F_{L}} & =\chi_{[2,(1,0)]}(\mathcal{D} ; \alpha, \beta), \\
\chi_{F_{R}} & =\chi_{[2,(0,1)]}(\mathcal{D} ; \alpha, \beta), \\
\chi_{C_{L}} & =\chi_{[3,(2,0)]}(\mathcal{D} ; \alpha, \beta), \\
\chi_{C_{R}} & =\chi_{[3,(0,2)]}(\mathcal{D} ; \alpha, \beta) .
\end{aligned}
$$

We could have included the characters for the U(1) gauge group in electromagnetism, but, since both the scalars and the photons are neutral, their characters would be trivial.

The characters for the unitary conformal representations of interest are $[53,55,56,65]$

$$
\begin{aligned}
\chi_{[1,(0,0)]}(\mathcal{D} ; \alpha, \beta) & =\mathcal{D} P(\mathcal{D} ; \alpha, \beta)\left(1-\mathcal{D}^{2}\right), \\
\chi_{[3 / 2,(1 / 2,0)]}(\mathcal{D} ; \alpha, \beta) & =\mathcal{D}^{3 / 2} P(\mathcal{D} ; \alpha, \beta)\left[\chi_{(1 / 2,0)}(\alpha, \beta)-\mathcal{D} \chi_{(0,1 / 2)}(\alpha, \beta)\right], \\
\chi_{[3 / 2,(0,1 / 2)]}(\mathcal{D} ; \alpha, \beta) & =\mathcal{D}^{3 / 2} P(\mathcal{D} ; \alpha, \beta)\left[\chi_{(0,1 / 2)}(\alpha, \beta)-\mathcal{D} \chi_{(1 / 2,0)}(\alpha, \beta)\right], \\
\chi_{[2,(1,0)]}(\mathcal{D} ; \alpha, \beta) & =\mathcal{D}^{2} P(\mathcal{D} ; \alpha, \beta)\left[\chi_{(1,0)}(\alpha, \beta)-\mathcal{D} \chi_{(1 / 2,1 / 2)}(\alpha, \beta)+\mathcal{D}^{2}\right], \\
\chi_{[2,(0,1)]}(\mathcal{D} ; \alpha, \beta) & =\mathcal{D}^{2} P(\mathcal{D} ; \alpha, \beta)\left[\chi_{(0,1)}(\alpha, \beta)-\mathcal{D} \chi_{(1 / 2,1 / 2)}(\alpha, \beta)+\mathcal{D}^{2}\right],
\end{aligned}
$$

\footnotetext{
${ }^{5}$ The modification term $\Delta \mathcal{H}$ will not be relevant for us as we consider operators with mass dimension greater than 4 .
} 


$$
\begin{aligned}
& \chi_{[3,(2,0)]}(\mathcal{D} ; \alpha, \beta)=\mathcal{D}^{3} P(\mathcal{D} ; \alpha, \beta)\left[\chi_{(2,0)}(\alpha, \beta)-\mathcal{D} \chi_{(3 / 2,1 / 2)}(\alpha, \beta)+\mathcal{D}^{2} \chi_{(1,0)}(\alpha, \beta)\right] \\
& \chi_{[3,(0,2)]}(\mathcal{D} ; \alpha, \beta)=\mathcal{D}^{3} P(\mathcal{D} ; \alpha, \beta)\left[\chi_{(0,2)}(\alpha, \beta)-\mathcal{D} \chi_{(1 / 2,3 / 2)}(\alpha, \beta)+\mathcal{D}^{2} \chi_{(0,1)}(\alpha, \beta)\right],
\end{aligned}
$$

where

$$
P(\mathcal{D} ; \alpha, \beta)=\frac{1}{(1-\mathcal{D} \alpha \beta)(1-\mathcal{D} /(\alpha \beta))(1-\mathcal{D} \alpha / \beta)(1-\mathcal{D} \beta / \alpha)}
$$

is the momentum generating function [53]. The characters of the Euclidean Lorentz group are simply products of $\mathrm{SU}(2)$ characters;

$$
\chi_{\left(l_{1}, l_{2}\right)}(\alpha, \beta)=\chi_{l_{1}}^{\mathrm{SU}(2)}(\alpha) \times \chi_{l_{2}}^{\mathrm{SU}(2)}(\beta) .
$$

The $\mathrm{SU}(2)$ characters we need are

$$
\begin{aligned}
& \chi_{0}^{\mathrm{SU}(2)}(\alpha)=1 \\
& \chi_{1 / 2}^{\mathrm{SU}(2)}(\alpha)=\alpha+\frac{1}{\alpha}, \\
& \chi_{1}^{\mathrm{SU}(2)}(\alpha)=\alpha^{2}+1+\frac{1}{\alpha^{2}}, \\
& \chi_{3 / 2}^{\mathrm{SU}(2)}(\alpha)=\alpha^{3}+\alpha+\frac{1}{\alpha}+\frac{1}{\alpha^{3}}, \\
& \chi_{2}^{\mathrm{SU}(2)}(\alpha)=\alpha^{4}+\alpha^{2}+1+\frac{1}{\alpha^{2}}+\frac{1}{\alpha^{4}} .
\end{aligned}
$$

Finally, the Haar measure for the Euclidean Lorentz group $\mathrm{SO}(4) \simeq \mathrm{SU}(2)_{L} \times \mathrm{SU}(2)_{R}$ is

$$
\int d \mu_{\text {Lorentz }}=\left(\frac{1}{2 \pi i}\right)^{2} \oint_{|\alpha|=1} \frac{d \alpha}{2 \alpha}\left(1-\alpha^{2}\right)\left(1-\frac{1}{\alpha^{2}}\right) \oint_{|\beta|=1} \frac{d \beta}{2 \beta}\left(1-\beta^{2}\right)\left(1-\frac{1}{\beta^{2}}\right) .
$$

\section{B Redundant operators}

The operator basis for leading-PM tidal effects in eq. (2.13) is a complete, non-redundant basis for all operators involving two scalars and two Weyl tensors. However, the explicit form of the operator basis involves some choices, originating from two types of redundancies: field redefinitions and integration-by-parts relations.

First we consider redundancies from field redefinitions. The free equation of motion (EOM) for the scalar field is

$$
\partial^{2} \phi+m^{2} \phi=0
$$

A composite operator which contains the factor $\partial^{2} \phi$ can be removed from the operator basis by an appropriate choice of field redefinition which exchanges it for the operator $m^{2} \phi$. 
When constructing operators where partial derivatives are acting on the scalar fields, we need only consider symmetric, traceless combinations of the derivatives. ${ }^{6}$

Similarly, the free EOM for the gauge field is

$$
\partial_{\mu} F^{\mu \nu}=0 .
$$

Also, we find that

$$
\partial^{2} F_{\mu \nu}=0
$$

by using the Bianchi identity $\partial_{[\alpha} F_{\mu \nu]}=0$. Again, we only need symmetric, traceless combinations of derivatives acting on the field strengths, where none of the derivatives are contracted with that field strength.

For gravity, we have Einstein's equation in vacuum:

$$
R_{\mu \nu}=0
$$

where $R_{\mu \nu}$ is the Ricci tensor. This means that we don't include the Ricci tensor nor the Ricci scalar in the operator basis as they can be removed by an appropriate redefinition of the metric tensor. ${ }^{7}$

Since the Weyl tensor is the traceless part of the Riemann tensor,

$$
C_{\mu \nu \rho \sigma}=R_{\mu \nu \rho \sigma}-\left(g_{\mu[\rho} R_{\sigma] \nu}-g_{\nu[\rho} R_{\sigma] \mu}\right)+\frac{1}{3} g_{\mu[\rho} g_{\sigma] \nu} R
$$

where $A_{[\mu \nu]}=\frac{1}{2}\left(A_{\mu \nu}-A_{\nu \mu}\right)$ for any tensor $A$, we can freely work with either the Riemann tensor or the Weyl tensor. For our purposes, it is most convenient to work with the Weyl tensor because it transforms in an irreducible representation of the Euclidean Lorentz group; see appendix A.

In vacuum, we find that

$$
\begin{aligned}
& \nabla^{\mu} C_{\mu \nu \rho \sigma}=0, \\
& \nabla^{2} C_{\mu \nu \rho \sigma}=\mathcal{O}\left(C^{2}\right),
\end{aligned}
$$

up to terms with Ricci tensors or Ricci scalars. Thus we need only keep symmetric, traceless combinations of covariant derivatives acting on the Weyl tensors.

Next we will illustrate the redundancies coming from integration-by-parts relations by looking at some possible dimension- 8 operators:

$$
\begin{aligned}
& \mathcal{O}_{1}=\phi \phi\left[\nabla_{\mu} C_{\rho \sigma \alpha \beta}\right]\left[\nabla^{\mu} C^{\rho \sigma \alpha \beta}\right], \\
& \mathcal{O}_{2}=\left[\nabla_{\mu} \phi\right]\left[\nabla^{\mu} \phi\right] C_{\rho \sigma \alpha \beta} C^{\rho \sigma \alpha \beta} \\
& \mathcal{O}_{3}=\phi\left[\nabla^{\mu} \phi\right]\left[\nabla_{\mu} C_{\rho \sigma \alpha \beta}\right] C^{\rho \sigma \alpha \beta} .
\end{aligned}
$$

\footnotetext{
${ }^{6}$ We could also replace the partial derivatives with covariant derivatives. Note that commutators of covariant derivatives are related to field strengths or curvature; $\left[D_{\mu}, D_{\nu}\right] \sim F_{\mu \nu}$ or $\left[\nabla_{\mu}, \nabla_{\nu}\right] \sim R$.

${ }^{7}$ From an amplitude perspective, ref. [66] showed that the modification of the Einstein-Hilbert action by the addition of $R^{2}$ and $R^{\mu \nu} R_{\mu \nu}$ terms does not affect the amplitude. Ref. [47] found an explicit field redefinition of the graviton field that removes traces of the Riemann curvature from the tidal worldline action, including in the presence of matter.
} 
Here, $\mathcal{O}_{1}$ corresponds to the operator with coefficient $c_{0}^{(1)}$ in eq. (2.13), while $\mathcal{O}_{2}$ and $\mathcal{O}_{3}$ are absent from eq. (2.13). The Hilbert series in eq. (2.4) informs us that there should be only one P-even operator at this mass dimension, but it does not tell us which one we should choose.

In fact, these operators are related through integration-by-parts relations,

$$
\begin{aligned}
\mathcal{O}_{1} & =\mathcal{O}_{2}+\mathrm{EOM}+\mathcal{O}\left(C^{3}\right), \\
\mathcal{O}_{1} & =-\mathcal{O}_{3},
\end{aligned}
$$

up to a total derivative, operators proportional to the leading-order EOM, and operators with more than two Weyl tensors. We discard the total derivative due to momentum conservation, and the EOM operators can be removed through a field redefinition. In fact, the Hilbert series have implicitly removed, whenever possible, operators with more derivatives in place of operators with fewer derivatives, i.e. using the EOM.

When we have more than one operator at a given mass dimension, we must carefully include independent operators which cannot be related through integration-by-parts relations or field redefinitions. A systematic way of taking into account integration-by-parts relations is detailed in refs. $[52,67]$. We enumerate all the ways of partitioning the derivatives (ignoring integration-by-parts relations), which we call $\left\{x_{i}\right\}$. Then we enumerate all gauge-invariant operators with one fewer covariant derivative which transform as a Lorentz four-vector, $\left\{y_{i}\right\}$. We can then apply a total derivative to the $y_{i}$ 's, which will generate a relation among the $x_{i}$ 's. The number of independent constraints coming from this procedure is given by the rank of the matrix of constraint equations.

Let's illustrate the procedure for the dimension- 8 operators. We assign the $x_{i}=\mathcal{O}_{i}$ for $i=1,2,3$. For the operators with one covariant derivative, we can have the covariant derivative act on a scalar or on a Weyl tensor;

$$
\begin{aligned}
& y_{1, \mu}=\phi\left[\nabla_{\mu} \phi\right] C_{\rho \sigma \alpha \beta} C^{\rho \sigma \alpha \beta}, \\
& y_{2, \mu}=\phi \phi\left[\nabla_{\mu} C_{\rho \sigma \alpha \beta}\right] C^{\rho \sigma \alpha \beta} .
\end{aligned}
$$

Now we apply the total derivative on the $y_{i}$ 's:

$$
\begin{aligned}
& \nabla^{\mu} y_{1, \mu}=x_{1}+2 x_{2}=0, \\
& \nabla^{\mu} y_{2, \mu}=2 x_{2}+x_{3}=0 .
\end{aligned}
$$

Note that we have dropped operators with $D^{2} \phi$ or $D^{2} C$ because they can either be removed by field redefinitions or produce operators with more than two Weyl tensors. We can write the equations in matrix form,

$$
M \cdot x \equiv\left(\begin{array}{lll}
1 & 2 & 0 \\
0 & 2 & 1
\end{array}\right)\left(\begin{array}{l}
x_{1} \\
x_{2} \\
x_{3}
\end{array}\right)=0 .
$$

The number of independent operators is $3-\operatorname{rank}(M)=3-2=1$. 
We have applied this method to ensure the operators in our basis are independent up to mass dimension 14. For the higher mass dimensions, we used the on-shell methods discussed in appendix C.

We have illustrated the freedom in choosing an operator basis coming from integrationby-parts relations. However, certain operator bases are better suited for calculations in the heavy limit. For example, the heavy limit of $\mathcal{O}_{2}$ feeds down to the dimension-6 operator $\phi \phi C_{\rho \sigma \alpha \beta} C^{\rho \sigma \alpha \beta}$, so this operator doesn't contribute new information with regards to the classical portion of the amplitude. In fact, we would also need to include subleading-in- $\hbar$ corrections from $\mathcal{O}_{2}$ to reproduce the correct subleading tidal effects.

The operator basis in eq. (2.13) is chosen to optimally produce all leading-PM tidal effects in the classical limit.

\section{Operator basis from an on-shell perspective}

A different approach to constructing the operator basis is to first look at the corresponding on-shell amplitudes. Following the discussion in ref. [68], we consider the non-factorizable part of the two-scalar-two-photon amplitude $\mathcal{A}(\phi \phi ; \gamma \gamma)$. We label the momenta for the photons by $p_{1}$ and $p_{2}$, and the momenta of the massive scalars by $p_{3}$ and $p_{4}$. For the helicity assignments $\gamma^{+}\left(p_{1}\right) \gamma^{+}\left(p_{2}\right)$ and $\gamma^{-}\left(p_{1}\right) \gamma^{+}\left(p_{2}\right)$, the structures carrying the correct little group weights are

[12] and $\left\langle 1\left|\left(p_{3}-p_{4}\right)\right| 2\right]$,

respectively. The amplitudes for the other helicity assignments can be constructed from the same building blocks after exchanging angle and square brackets. The non-factorizable part of the two amplitudes with positive helicity for $p_{2}$ are

$$
\begin{aligned}
& \mathcal{A}\left(\phi \phi ; \gamma^{+}\left(p_{1}\right), \gamma^{+}\left(p_{2}\right)\right)=[12]^{2} a\left(s_{12}, s_{13}, s_{14}\right), \\
& \mathcal{A}\left(\phi \phi ; \gamma^{-}\left(p_{1}\right), \gamma^{+}\left(p_{2}\right)\right)=\left\langle 1\left|\left(p_{3}-p_{4}\right)\right| 2\right]^{2} b\left(s_{12}, s_{13}, s_{14}\right),
\end{aligned}
$$

where $a\left(s_{12}, s_{13}, s_{14}\right)$ and $b\left(s_{12}, s_{13}, s_{14}\right)$ are polynomials of the Mandelstam variables $s_{i j}=$ $\left(p_{i}+p_{j}\right)^{2}$. Taking into account the relation $s_{12}+s_{13}+s_{14}=2 m^{2}$, and keeping the symmetry $3 \leftrightarrow 4$, the polynomials take the form

$$
\begin{aligned}
& a\left(s_{12}, s_{13}, s_{14}\right)=\sum_{i=0}^{\infty} \sum_{j=0}^{\infty} \frac{a_{i, j}}{\Lambda^{2 i+4 j+2}} s_{12}^{i}\left(s_{13} s_{14}\right)^{j}, \\
& b\left(s_{12}, s_{13}, s_{14}\right)=\sum_{i=0}^{\infty} \sum_{j=0}^{\infty} \frac{b_{i, j}}{\Lambda^{2 i+4 j+4}} s_{12}^{i}\left(s_{13} s_{14}\right)^{j},
\end{aligned}
$$

where $\Lambda$ is some unfixed dimensionful scale and $a_{i, j}$ and $b_{i, j}$ are dimensionless coefficients.

By comparing the non-factorizable part of the on-shell amplitudes with the output of the Hilbert series in eq. (2.2), one can find a correspondence between the Wilson coefficients of the action and the coefficients $a_{i, j}, b_{i, j}$. This helps us in inferring the higher-dimensional operators, since we can now construct operators which have the field content given by the Hilbert series and which reduce to the amplitudes when imposing on-shell conditions. 
Similarly, we can compare the on-shell amplitudes for two scalars and two gravitons,

$$
\begin{aligned}
& \mathcal{M}\left(\phi \phi ; g^{2+}\left(p_{1}\right), g^{2+}\left(p_{2}\right)\right)=[12]^{4} c\left(s_{12}, s_{13}, s_{14}\right), \\
& \mathcal{M}\left(\phi \phi ; g^{2-}\left(p_{1}\right), g^{2+}\left(p_{2}\right)\right)=\left\langle 1\left|\left(p_{3}-p_{4}\right)\right| 2\right]^{4} d\left(s_{12}, s_{13}, s_{14}\right),
\end{aligned}
$$

with the output of the Hilbert series in eq. (2.4). The same arguments apply to the polynomials $c$ and $d$ as $a$ and $b$, so they become

$$
\begin{aligned}
& c\left(s_{12}, s_{13}, s_{14}\right)=\sum_{i=0}^{\infty} \sum_{j=0}^{\infty} \frac{c_{i, j}}{\Lambda^{2 i+4 j+4}} s_{12}^{i}\left(s_{13} s_{14}\right)^{j}, \\
& d\left(s_{12}, s_{13}, s_{14}\right)=\sum_{i=0}^{\infty} \sum_{j=0}^{\infty} \frac{d_{i, j}}{\Lambda^{2 i+4 j+8}} s_{12}^{i}\left(s_{13} s_{14}\right)^{j},
\end{aligned}
$$

with dimensionless coefficients $c_{i, j}$ and $d_{i, j}$. We see a similar correspondence between the on-shell amplitudes and the effective operators as in the QED case.

Open Access. This article is distributed under the terms of the Creative Commons Attribution License (CC-BY 4.0), which permits any use, distribution and reproduction in any medium, provided the original author(s) and source are credited.

\section{References}

[1] B.S. DeWitt, Quantum Theory of Gravity. 2. The Manifestly Covariant Theory, Phys. Rev. 162 (1967) 1195 [INSPIRE].

[2] Y. Iwasaki, Quantum theory of gravitation vs. classical theory: fourth-order potential, Prog. Theor. Phys. 46 (1971) 1587 [inSPIRE].

[3] J.F. Donoghue, Leading quantum correction to the Newtonian potential, Phys. Rev. Lett. 72 (1994) 2996 [gr-qc/9310024] [INSPIRE].

[4] J.F. Donoghue, General relativity as an effective field theory: The leading quantum corrections, Phys. Rev. D 50 (1994) 3874 [gr-qc/9405057] [INSPIRE].

[5] N.E.J. Bjerrum-Bohr, J.F. Donoghue and B.R. Holstein, Quantum gravitational corrections to the nonrelativistic scattering potential of two masses, Phys. Rev. D 67 (2003) 084033 [Erratum ibid. 71 (2005) 069903] [hep-th/0211072] [INSPIRE].

[6] B.R. Holstein and A. Ross, Spin Effects in Long Range Gravitational Scattering, arXiv: 0802.0716 [INSPIRE].

[7] D. Neill and I.Z. Rothstein, Classical Space-Times from the S Matrix, Nucl. Phys. B $\mathbf{8 7 7}$ (2013) 177 [arXiv:1304.7263] [INSPIRE].

[8] N.E.J. Bjerrum-Bohr, J.F. Donoghue and P. Vanhove, On-shell Techniques and Universal Results in Quantum Gravity, JHEP 02 (2014) 111 [arXiv:1309.0804] [INSPIRE].

[9] V. Vaidya, Gravitational spin Hamiltonians from the S matrix, Phys. Rev. D 91 (2015) 024017 [arXiv: 1410 . 5348] [INSPIRE].

[10] N.E.J. Bjerrum-Bohr, J.F. Donoghue and B.R. Holstein, Quantum corrections to the Schwarzschild and Kerr metrics, Phys. Rev. D 68 (2003) 084005 [Erratum ibid. 71 (2005) 069904] [hep-th/0211071] [INSPIRE]. 
[11] LIGO Scientific and Virgo collaborations, Observation of Gravitational Waves from a Binary Black Hole Merger, Phys. Rev. Lett. 116 (2016) 061102 [arXiv:1602.03837] [INSPIRE].

[12] B. Bertotti, On gravitational motion, Nuovo Cim. 4 (1956) 898 [INSPIRE].

[13] B. Bertotti and J. Plebanski, Theory of gravitational perturbations in the fast motion approximation, Ann. Phys. 11 (1960) 169 [INSPIRE].

[14] C. Cheung, I.Z. Rothstein and M.P. Solon, From Scattering Amplitudes to Classical Potentials in the Post-Minkowskian Expansion, Phys. Rev. Lett. 121 (2018) 251101 [arXiv: 1808.02489] [INSPIRE].

[15] A. Cristofoli, N.E.J. Bjerrum-Bohr, P.H. Damgaard and P. Vanhove, Post-Minkowskian Hamiltonians in general relativity, Phys. Rev. D 100 (2019) 084040 [arXiv:1906.01579] [INSPIRE].

[16] Z. Bern, A. Luna, R. Roiban, C.-H. Shen and M. Zeng, Spinning Black Hole Binary Dynamics, Scattering Amplitudes and Effective Field Theory, arXiv:2005.03071 [INSPIRE].

[17] D.A. Kosower, B. Maybee and D. O'Connell, Amplitudes, Observables, and Classical Scattering, JHEP 02 (2019) 137 [arXiv:1811.10950] [INSPIRE].

[18] B. Maybee, D. O'Connell and J. Vines, Observables and amplitudes for spinning particles and black holes, JHEP 12 (2019) 156 [arXiv:1906.09260] [INSPIRE].

[19] G. Kälin and R.A. Porto, From Boundary Data to Bound States, JHEP 01 (2020) 072 [arXiv: 1910.03008] [INSPIRE].

[20] N.E.J. Bjerrum-Bohr, A. Cristofoli and P.H. Damgaard, Post-Minkowskian Scattering Angle in Einstein Gravity, JHEP 08 (2020) 038 [arXiv: 1910.09366] [INSPIRE].

[21] A. Cristofoli, P.H. Damgaard, P. Di Vecchia and C. Heissenberg, Second-order Post-Minkowskian scattering in arbitrary dimensions, JHEP 07 (2020) 122 [arXiv:2003.10274] [INSPIRE].

[22] A. Cristofoli, Gravitational shock waves and scattering amplitudes, JHEP 11 (2020) 160 [arXiv:2006.08283] [INSPIRE].

[23] Z. Bern, C. Cheung, R. Roiban, C.-H. Shen, M.P. Solon and M. Zeng, Scattering Amplitudes and the Conservative Hamiltonian for Binary Systems at Third Post-Minkowskian Order, Phys. Rev. Lett. 122 (2019) 201603 [arXiv:1901.04424] [inSPIRE].

[24] Z. Bern, C. Cheung, R. Roiban, C.-H. Shen, M.P. Solon and M. Zeng, Black Hole Binary Dynamics from the Double Copy and Effective Theory, JHEP 10 (2019) 206 [arXiv: 1908.01493] [INSPIRE].

[25] C. Cheung and M.P. Solon, Classical gravitational scattering at $\mathcal{O}\left(G^{3}\right)$ from Feynman diagrams, JHEP 06 (2020) 144 [arXiv:2003.08351] [INSPIRE].

[26] C. Cheung and M.P. Solon, Tidal Effects in the Post-Minkowskian Expansion, Phys. Rev. Lett. 125 (2020) 191601 [arXiv:2006.06665] [INSPIRE].

[27] Z. Bern, H. Ita, J. Parra-Martinez and M.S. Ruf, Universality in the classical limit of massless gravitational scattering, Phys. Rev. Lett. 125 (2020) 031601 [arXiv:2002.02459] [INSPIRE]. 
[28] A. Brandhuber and G. Travaglini, On higher-derivative effects on the gravitational potential and particle bending, JHEP 01 (2020) 010 [arXiv: 1905.05657] [INSPIRE].

[29] A. Cristofoli, Post-Minkowskian Hamiltonians in Modified Theories of Gravity, Phys. Lett. B 800 (2020) 135095 [arXiv: 1906.05209] [INSPIRE].

[30] P.H. Damgaard, K. Haddad and A. Helset, Heavy Black Hole Effective Theory, JHEP 11 (2019) 070 [arXiv: 1908.10308] [INSPIRE].

[31] A. Guevara, Holomorphic Classical Limit for Spin Effects in Gravitational and Electromagnetic Scattering, JHEP 04 (2019) 033 [arXiv: 1706.02314] [INSPIRE].

[32] N. Arkani-Hamed, T.-C. Huang and Y.-t. Huang, Scattering Amplitudes For All Masses and Spins, arXiv:1709.04891 [INSPIRE].

[33] A. Guevara, A. Ochirov and J. Vines, Scattering of Spinning Black Holes from Exponentiated Soft Factors, JHEP 09 (2019) 056 [arXiv: 1812.06895] [INSPIRE].

[34] M.-Z. Chung, Y.-T. Huang, J.-W. Kim and S. Lee, The simplest massive S-matrix: from minimal coupling to Black Holes, JHEP 04 (2019) 156 [arXiv: 1812.08752] [INSPIRE].

[35] M.-Z. Chung, Y.-T. Huang and J.-W. Kim, Classical potential for general spinning bodies, JHEP 09 (2020) 074 [arXiv: 1908.08463] [InSPIRE].

[36] A. Guevara, A. Ochirov and J. Vines, Black-hole scattering with general spin directions from minimal-coupling amplitudes, Phys. Rev. D 100 (2019) 104024 [arXiv:1906.10071] [INSPIRE].

[37] N. Arkani-Hamed, Y.-t. Huang and D. O'Connell, Kerr black holes as elementary particles, JHEP 01 (2020) 046 [arXiv:1906.10100] [INSPIRE].

[38] R. Aoude, K. Haddad and A. Helset, On-shell heavy particle effective theories, JHEP 05 (2020) 051 [arXiv : 2001.09164] [INSPIRE].

[39] M.-Z. Chung, Y.-t. Huang, J.-W. Kim and S. Lee, Complete Hamiltonian for spinning binary systems at first post-Minkowskian order, JHEP 05 (2020) 105 [arXiv: 2003.06600] [InSPIRE].

[40] R. Aoude, M.-Z. Chung, Y.-t. Huang, C.S. Machado and M.-K. Tam, Silence of Binary Kerr Black Holes, Phys. Rev. Lett. 125 (2020) 181602 [arXiv: 2007.09486] [INSPIRE].

[41] T. Damour, M. Soffel and C.-m. Xu, General relativistic celestial mechanics. 3. Rotational equations of motion, Phys. Rev. D 47 (1993) 3124 [INSPIRE].

[42] T. Damour and A. Nagar, Effective One Body description of tidal effects in inspiralling compact binaries, Phys. Rev. D 81 (2010) 084016 [arXiv:0911.5041] [INSPIRE].

[43] A. Buonanno and T. Damour, Effective one-body approach to general relativistic two-body dynamics, Phys. Rev. D 59 (1999) 084006 [gr-qc/9811091] [INSPIRE].

[44] D. Bini, T. Damour and G. Faye, Effective action approach to higher-order relativistic tidal interactions in binary systems and their effective one body description, Phys. Rev. D $\mathbf{8 5}$ (2012) 124034 [arXiv:1202.3565] [INSPIRE].

[45] D. Bini, T. Damour and A. Geralico, Scattering of tidally interacting bodies in post-Minkowskian gravity, Phys. Rev. D 101 (2020) 044039 [arXiv: 2001.00352] [InSPIRE].

[46] G. Kälin and R.A. Porto, Post-Minkowskian Effective Field Theory for Conservative Binary Dynamics, JHEP 11 (2020) 106 [arXiv:2006.01184] [INSPIRE]. 
[47] Q. Henry, G. Faye and L. Blanchet, Tidal effects in the equations of motion of compact binary systems to next-to-next-to-leading post-Newtonian order, Phys. Rev. D 101 (2020) 064047 [arXiv: 1912.01920] [INSPIRE].

[48] S. Benvenuti, B. Feng, A. Hanany and Y.-H. He, Counting BPS Operators in Gauge Theories: Quivers, Syzygies and Plethystics, JHEP 11 (2007) 050 [hep-th/0608050] [INSPIRE].

[49] B. Feng, A. Hanany and Y.-H. He, Counting gauge invariants: The Plethystic program, JHEP 03 (2007) 090 [hep-th/0701063] [INSPIRE].

[50] E.E. Jenkins and A.V. Manohar, Algebraic Structure of Lepton and Quark Flavor Invariants and CP-violation, JHEP 10 (2009) 094 [arXiv: 0907.4763] [INSPIRE].

[51] L. Lehman and A. Martin, Hilbert Series for Constructing Lagrangians: expanding the phenomenologist's toolbox, Phys. Rev. D 91 (2015) 105014 [arXiv:1503.07537] [INSPIRE].

[52] L. Lehman and A. Martin, Low-derivative operators of the Standard Model effective field theory via Hilbert series methods, JHEP 02 (2016) 081 [arXiv: 1510.00372] [INSPIRE].

[53] B. Henning, X. Lu, T. Melia and H. Murayama, 2, 84, 30, 993, 560, 15456, 11962, 261485, ...: Higher dimension operators in the SM EFT, JHEP 08 (2017) 016 [Erratum JHEP 09 (2019) 019] [arXiv: 1512.03433] [INSPIRE].

[54] B. Henning, X. Lu, T. Melia and H. Murayama, Hilbert series and operator bases with derivatives in effective field theories, Commun. Math. Phys. 347 (2016) 363 [arXiv: 1507.07240] [INSPIRE].

[55] B. Henning, X. Lu, T. Melia and H. Murayama, Operator bases, S-matrices, and their partition functions, JHEP 10 (2017) 199 [arXiv:1706.08520] [INSPIRE].

[56] M. Ruhdorfer, J. Serra and A. Weiler, Effective Field Theory of Gravity to All Orders, JHEP 05 (2020) 083 [arXiv: 1908.08050] [INSPIRE].

[57] Z. Bern, J. Parra-Martinez, R. Roiban, E. Sawyer and C.-H. Shen, Leading Nonlinear Tidal Effects and Scattering Amplitudes, arXiv:2010.08559 [INSPIRE].

[58] N.J. Poplawski, Classical Physics: Spacetime and Fields, arXiv:0911.0334 [InSPIRE].

[59] A.H. Guth, M.P. Hertzberg and C. Prescod-WEinstein, Do Dark Matter Axions Form a Condensate with Long-Range Correlation?, Phys. Rev. D 92 (2015) 103513 [arXiv: 1412.5930] [INSPIRE].

[60] M.H. Namjoo, A.H. Guth and D.I. Kaiser, Relativistic Corrections to Nonrelativistic Effective Field Theories, Phys. Rev. D 98 (2018) 016011 [arXiv:1712.00445] [INSPIRE].

[61] E. Braaten, A. Mohapatra and H. Zhang, Classical Nonrelativistic Effective Field Theories for a Real Scalar Field, Phys. Rev. D 98 (2018) 096012 [arXiv: 1806. 01898] [INSPIRE].

[62] K. Haddad and A. Helset, Relating Gauge and Gravity Theories in the Large Mass Limit, Phys. Rev. Lett. 125 (2020) 181603 [arXiv:2005.13897] [InSPIRE].

[63] G. Passarino and M.J.G. Veltman, One Loop Corrections for $e^{+} e^{-}$Annihilation Into $\mu^{+} \mu^{-}$ in the Weinberg Model, Nucl. Phys. B 160 (1979) 151 [INSPIRE].

[64] W.D. Goldberger and I.Z. Rothstein, An Effective field theory of gravity for extended objects, Phys. Rev. D 73 (2006) 104029 [hep-th/0409156] [INSPIRE]. 
[65] A. Barabanschikov, L. Grant, L.L. Huang and S. Raju, The Spectrum of Yang-Mills on a sphere, JHEP 01 (2006) 160 [hep-th/0501063] [INSPIRE].

[66] M. Accettulli Huber, A. Brandhuber, S. De Angelis and G. Travaglini, Note on the absence of $R^{2}$ corrections to Newton's potential, Phys. Rev. D 101 (2020) 046011 [arXiv: 1911.10108] [INSPIRE].

[67] C. Hays, A. Martin, V. Sanz and J. Setford, On the impact of dimension-eight SMEFT operators on Higgs measurements, JHEP 02 (2019) 123 [arXiv: 1808.00442] [INSPIRE].

[68] Y. Shadmi and Y. Weiss, Effective Field Theory Amplitudes the On-Shell Way: Scalar and Vector Couplings to Gluons, JHEP 02 (2019) 165 [arXiv: 1809.09644] [INSPIRE]. 